\title{
Combined hormonal contraceptives and the risk of venous and arterial thromboembolism and cardiovascular death: misuse of automated databases
}

\author{
Samuel Shapiro
}

Visiting Professor of Epidemiology, Department of Family Medicine and Public Health, University of Cape Town School of Medicine, Cape Town, South Africa

\section{Correspondence to} Professor Samuel Shapiro, Department of Family Medicine and Public Health, University of Cape Town School of Medicine, Anzio Road, Observatory, Cape Town, South Africa: samshap@mweb.co.za

Received 31 December 2012 Revised 5 February 2013 Accepted 5 February 2013
To cite: Shapiro S. Journal of Family Planning and Reproductive Health Care 2013;39:89-96.

\begin{abstract}
Background In December 2011, the US Food and Drug Administration (FDA) convened a public Advisory Committee meeting to review evidence from a study commissioned by the agency. An analysis of findings derived from four databases was published on the FDA website, and presented at the meeting. Among users of combined hormonal contraceptives containing ethinylestradiol (EE) plus drospirenone (DRSP) the risks of venous (VTE) and arterial thromboembolism (ATE) were higher than among users of older reference contraceptives containing other progestogens. The findings have now been published in a peer-reviewed journal.

Objective To evaluate the published evidence. Methods Generally accepted epidemiological principles of causality are applied.

Results The findings did not satisfy the criteria of time order, bias, confounding, statistical stability and strength of association, durationresponse, internal consistency, external consistency, or biological plausibility.

Conclusions The best evidence continues to suggest that the increased risk of VTE in combined hormonal contraceptive users is dependent on the dose of estrogen, and independent of the progestogen used. The best evidence also suggests that DRSP does not increase the risk of ATE, and may reduce it.
\end{abstract}

\section{BACKGROUND}

In December 2011, the US Food and Drug Administration (FDA) convened a public Advisory Committee meeting to consider new findings regarding the risk of venous thromboembolism (VTE), and of arterial thromboembolism (ATE) [myocardial infarction (MI) and stroke combined], in users of recently introduced combined estrogen/progestogen hormonal contraceptives (CHCs). ${ }^{1}$ At the time of the meeting the findings had only been published on the FDA website, but not in a peer-reviewed journal.

The investigators concluded that their data "[provided] another positive finding to the increasing body of evidence linking [drospirenone (DRSP)] to increased risk of VTE relative to standard low-dose CHC pills. DRSP was associated with higher risk of ATE in new users overall with this finding restricted to women in the 35-55 years age group only". Those conclusions were then invoked in the medical literature as grounds for public health recommendations. ${ }^{2}$

In November 2012, the FDA findings for VTE, ATE and total mortality were published in a peer-reviewed journal, ${ }^{3}$ and here they are evaluated.

\section{SIDNEY ET AL. $2012^{3}$}

Automated prescription and diagnostic data were derived from four sites: Kaiser Permanente Northern California, Kaiser Permanente Southern California, Tennessee State Medicaid and Washington State Medicaid. Mortality data were obtained from state registries. Women aged 1055 years with a computer record of at least one CHC prescription between 2001 and 2007 were studied.

'New users' of CHCs were defined as women not previously exposed to any CHC during the 2001-2007 study period. In addition, women exposed 
during the first 6 months of 2001 were only defined as new users if they had not been exposed in the preceding 6 months. Users of DRSP $(3.0 \mathrm{mg}) /$ ethinylestradiol (EE) $(30 \mu \mathrm{g})$ tablets, the norgestromin $(6.0 \mathrm{mg}) / \mathrm{EE}$ $(750 \mu \mathrm{g})$ patch and the etonogestrel $(11.7 \mathrm{mg}) / \mathrm{EE}$ $2700 \mu \mathrm{g}$ vaginal ring were compared with a reference category of users of "four low-dose estrogen comparator CHCs" [levonorgestrel $(0.10 \mathrm{mg}) / \mathrm{EE} \quad(20 \mu \mathrm{g})$ tablets; levonorgestrel $(0.15 \mathrm{mg}) / \mathrm{EE}(30 \mu \mathrm{g})$ tablets; norethindrone $(1 \mathrm{mg}) / \mathrm{EE}(20 \mathrm{mg})$ tablets; norgestimate $(0.18-0.25 \mathrm{mg}) / \mathrm{EE}(35 \mu \mathrm{g})$ tablets].

Hospitalised cases of VTE, as well as cases of ATE, were validated by a panel of physicians kept unaware of the exposure status of the patient. Outpatient cases of VTE had received anticoagulants for at least 30 days.

Relative risks (RRs) adjusted for age, site, and year of entry into the study were estimated. A total of 12 additional factors prevalent in $\geq 1 \%$ of the cohorts [angiotensin-converting-enzyme (ACE) inhibitors; acne; asthma; $\beta$-blockers; diabetes, hormone replacement therapy, hyperlipidaemia; hypertension; migraine; non-steroidal anti-inflammatory drugs (NSAIDs); statins; surgery/injury] were evaluated as potential confounders. In the analysis of VTE risk the additional factors did not materially confound the comparisons, and they were not included in the statistical models; in the analysis of ATE risk, and of the risk of total mortality, hypertension, hyperlipidaemia and diabetes were also included in the models.

There were 573680 new CHC users. Overall, the respective confounder-adjusted incidence rates of VTE in DRSP users (74 cases), and in the reference category (205 cases), were 13.7 and 8.2 per 10000 person years (PY), and the adjusted RR was 1.77 [95\% confidence interval (CI) 1.33-2.35]. For ATE the corresponding incidence rates were 2.5 (14 cases) and 1.8 (45 cases) per $10000 \mathrm{PY}$, and the RR was 2.01 (95\% CI 1.06-3.81). The increased risk of VTE was restricted to DRSP users aged 10-34 years (RR 2.12; 95\% CI 1.43-3.15). The increased risk of ATE was restricted to users aged $35-55$ years $(2.60 ; 95 \%$ CI 1.25-5.41). For durations of DRSP use of $<3,3-12$ (sic), and $>12$ (sic) months the respective RR estimates were 1.96, 1.88, and 1.29 for VTE, and 1.67, 1.67, and 2.74 for ATE. The RR of total mortality in DRSP users (17 cases) was 0.88 (95\% CI 0.52-1.53).

Among norgestromin and etonogestrel users there were no significant increases in the risks of VTE (33 and 9 cases, respectively), ATE ( 4 and 2 cases) or total mortality (13 and 3 cases).

\section{EVALUATION}

Among the norgestromin users there were 33 cases of VTE (RR 1.09; 95\% CI 0.30-1.69). For all other outcomes among users of norgestromin and etonogestrel the data were sparse (2-13 cases), and there were no 'signals' suggestive of an increased risk. Here the findings for those $\mathrm{CHCs}$ are not considered further. Below the focus is on the findings for DRSP, and generally accepted epidemiological principles of causation $^{4-7}$ are applied to the evidence.

\section{VENOUS THROMBOEMBOLISM}

Before applying causal criteria to the evidence it is helpful first to set the background. When CHCs were initially introduced, evaluation of the risk of VTE in $\mathrm{CHC}$ users and non-users proved to be relatively straightforward, ${ }^{8}$ principally because the increased risk (of the order of three-fold or more) was large. However, comparisons of the magnitude of the risk of VTE confined to users of different CHCs have proven not to be straightforward, for the following reasons.

1 CHCs, as a class, increase the risk of VTE, and the magnitude of the increase is dependent on the estrogen dose. ${ }^{8}$ That relationship is well established, and universally accepted. What is controversial, however, is whether, among women taking $\mathrm{CHCs}$ containing the same or a similar dose of $\mathrm{EE}$, combining $\mathrm{EE}$ with DRSP confers a higher risk than combining EE with other progestogens.

2 The severity of VTE varies from virtually no symptoms ('occult' VTE) at the one extreme, to death (almost entirely due to pulmonary embolism) at the other; the great majority of cases are mild (e.g. a swollen leg), and they recover fully. ${ }^{9}$ Thus, unless there are grounds for suspicion, occult cases can readily go undiagnosed, but can selectively be diagnosed in CHC users. In addition, such cases can more commonly be diagnosed if they are using a $\mathrm{CHC}$ currently given adverse publicity than if they are using a $\mathrm{CHC}$ not receiving publicity.

3 In recent years technical advances in the diagnosis of VTE (e.g. d-dimer testing, duplex and triplex ultrasonography, magnetic resonance imaging) have resulted in the detection of occult cases that previously escaped diagnosis. As a result, the documented incidence of VTE among women of reproductive age who are not taking CHCs has increased from about 1-2 to 4-5 per $10000 \mathrm{PY}^{10-14}$; among $\mathrm{CHC}$ users corresponding increases have taken place.

A further reason why the incidence of VTE has increased is because the prevalence of obesity has increased. ${ }^{15}$ Obesity substantially increases the risk of VTE, and the incidence will consequently have increased both among non-users and users of CHCs. In addition, the combined effects of age and obesity are more than additive. ${ }^{10-14}$

It follows that in a study in which the incidence of VTE is compared among users of two or more different CHCs, the increase over time makes it obligatory to compare women of the same age, who use CHCs over the same time intervals, and who commence use at the same time. Assume, for example, that DRSP users, who could not have commenced use before 2001 (the year of introduction) are compared with 
users of older products, who could have commenced decades earlier. Even if there is in fact no difference in the incidence of VTE, because of technical advances, as well as changes in the distribution of risk factors such as obesity, the incidence would appear to be higher in the DRSP users, and the comparison would be biased. Confounding would also occur, unless full allowance for the combined effects of age and obesity, and for other factors such as a family history of VTE, are made.

4 Consider two hypothetical women, one a DRSP user and one a user of an older CHC. Assume that both women present with a swollen leg, that both have a diagnosis of VTE, and that both recover. Because of the extensive publicity given to the DRSP/ VTE relationship, the DRSP user is more likely than the reference user to suspect VTE; she is more likely to consult her doctor; and her doctor is more likely to carry out diagnostic procedures, and correctly make the diagnosis. ${ }^{16}$

Whenever a new product is introduced, and whenever it is known, in advance, that a class effect such as VTE can occur, initially there is a selective tendency for doctors and others to report its occurrence more commonly among users of the newer product than of the older products in the medical literature, as well as to regulatory agencies, and to give it more publicity in the media. ${ }^{16}$

To illustrate: assume that two women are, respectively, users of DRSP and a reference CHC, and that both are diagnosed with VTE. Since DRSP is still relatively new, the user's VTE is published in a medical journal, and reported to a regulatory agency. However, the VTE in the user of the older CHC is neither published nor reported, since "everyone already knows, and has known for years, that CHCs cause VTE, so it is not necessary to report it once again". In addition, after a series of DRSP-induced cases have been published in the medical literature, adverse publicity in the media follows, whereas the same adverse publicity is not given to the older CHC.

From the perspective of validity, the bias due to differential reporting can be reduced, but not be eliminated, by ensuring that the same time intervals are compared. In addition, since bias cannot be eliminated entirely, if the risk for DRSP versus an older reference $\mathrm{CHC}$ appears only to be modestly increased, it may be impossible to judge whether the association is causal, or due to bias or confounding. ${ }^{17}$

5 For all $\mathrm{CHCs}$ as a class it is now established that, among women using CHCs for the first time ('starters') the risk of VTE is increased some four- to five-fold when use commences, within 3 months it declines to about two- to three-fold; then, over a span of years it continues slowly to decline. ${ }^{10-14} 1819$ When women stop using CHCs in order to become pregnant, or for other reasons, within a month or two the risk declines to that of non-users. And each time
CHC use is re-commenced ('re-starters'), the pattern of an initially marked increase in incidence, followed by a decline, repeats itself. But since the incidence of VTE rises sharply with increasing age, each successive pattern of an initially high risk, followed by a decline, is not at the same level. It is also possible that the magnitude of the pattern of increased risk differs for starters and re-starters.

The importance of this complex sequence cannot be sufficiently emphasised. From the perspective of making valid comparisons among users of DRSP versus any reference $\mathrm{CHC} / \mathrm{s}$, it is mandatory to take duration of use, and especially short-duration use, fully into account by comparing 1-3-month users with 1-3-month users, 6-11-month users with 611-month users, 1-2-year users with 1-2-year users, and so on. It is also mandatory to compare women who commence use at exactly the same time, and at the same age.

To illustrate: assume that the incidence of VTE is the same in DRSP and in reference CHC users. If 13 -month users of DRSP are compared with 611-month users of the reference product/s, the risk will nevertheless appear, spuriously, to be higher for DRSP users because the risk of VTE declines with increasing duration of use.

6 Computer-recorded CHC prescriptions are inherently unreliable. Some women may decide not to use a prescribed CHC. Some women who already have an existing supply may defer use, sometimes for months. Some women may stop because they wish to become pregnant. And some women stop simply because they do not need to use a CHC, and they may start again when they do.

Computer data on short-duration use are especially unreliable. ${ }^{19}$ Oral CHCs are usually prescribed for 2 or 3 months, and for women with only one or two recorded prescriptions it is impossible to be sure about the exact duration of use in months. Many women prescribed a CHC do not even start. Thus estimation of duration of use based solely on automated data results in overestimation of actual duration.

In order to accurately record the timing and duration of use, especially short-duration use, only information obtained directly from the woman herself can be considered reliable. In addition the data may be biased if, as is likely, computer-recorded shortduration use of a relatively new $\mathrm{CHC}$ tends to be shorter than computer-recorded short-duration use of an older product.

7 Epidemiology is not an exact science, and even in well-designed studies the possibility of bias or confounding cannot be entirely eliminated. ${ }^{17}$ If, however, a study is of high quality, and if the risk for users of one $\mathrm{CHC}$ is increased several-fold (say, five-fold or greater) relative to the use of another $\mathrm{CHC}$, it may be reasonable to judge that even if all sources of bias and 
confounding could indeed be eliminated, the association may be somewhat reduced (say, to three- or four-fold), but still be evident. Consequently it would be reasonable to conclude that, the limitations notwithstanding, the association is causal. However, when a risk is only modestly increased (say, less than two-fold), it may be impossible to discriminate among bias, confounding, and causation as alternative explanations. ${ }^{17}$

To sum up: in a valid comparison of VTE risk it is necessary to compare women who start using DRSP with women of the same age who also start using the reference $\mathrm{CHC}$ at the same time - and preferably to compare starters who have never before used a $\mathrm{CHC}$ ('first-time-ever' users) - or failing that, to compare first-time-ever-users, combined with women who only re-start after several years of non-use - and to compare the same durations of use. And even then, because bias and confounding cannot entirely be eliminated, it may be impossible to judge whether low-magnitude associations can be interpreted as causal.

I turn next to the application of causal principles ${ }^{4-7}$ to the evidence. ${ }^{3}$ The principles are inter-related (in particular, in the present instance, the criteria of time order and confounding), and when appropriate, I cross-refer.

\section{Time order}

During the first 6 months of follow-up in 2001, the definition of 'new use' of DRSP or of a reference $\mathrm{CHC}$ based on a 6-month exclusion period preceding 2001 was inadequate (see: Confounding). To illustrate: a woman who a year or two previously used a $\mathrm{CHC}$, and who stopped because she became obese and hypertensive, could have switched to condoms, and then, when she recommenced CHC use preferentially have been prescribed DRSP because of its antihypertensive action $^{20}$ - and much the same considerations would apply to women whose first recorded $\mathrm{CHC}$ use was during the second half of 2001, or during 2002, 2003, or later. Alternatively, general ill-health (e.g. asthma, arthritis) predisposes to VTE, and affected women could selectively have stopped using CHCs during exacerbations, and years later have re-started after having been prescribed DRSP in the (mistaken) belief that it was 'safer' than older CHCs (see: Confounding).

Violation of time order could only confidently have been reduced by confining the analysis to first-time-ever users - which based on automated data was not possible - or failing that, the exclusion interval should have been several years - which also was not possible.

\section{Detection bias}

From the time of its introduction in 2001, increasing publicity, including publicity in the USA from a large and increasing number of law suits, has been given to the allegation that DRSP causes more VTE than do older CHCs. Inevitably, therefore, there would have been a selective tendency to suspect and diagnose VTE more commonly in DRSP users than in users of the reference CHCs. The likelihood of detection bias was not mentioned in the published report. ${ }^{3}$

\section{Confounding}

The study lacked any information on obesity [body mass index (BMI)], family history of VTE and socioeconomic status, each of them established risk factors for VTE. DRSP tends preferentially to be prescribed to obese women, ${ }^{10}$ and the effects of obesity and age, both determinants of CHC use and of VTE risk, are more than additive ('interaction'). Analogous considerations apply to family history and socioeconomic status.

In one study ${ }^{10} 19$ in which full adjustment was made for age, BMI, duration of current use of DRSP or levonorgestrel (see below: Duration-response), family history, level of education (an index of socioeconomic status), and the interaction between BMI and age, the RR for DRSP versus the reference CHCs was reduced by $27 \%$ as compared with adjustment only for age. By extrapolation those findings imply that had it been possible to fully adjust for the relevant variables in the present study, ${ }^{3}$ the RR would have been reduced to about $1.29[1.77 \times(1.00-0.27)]$. That is, there would hardly have been a perceptible increase in the risk of VTE among DRSP users.

Confounding could also have occurred because diagnostic methods, diagnostic precision and referral patterns among users of different CHCs changed over the time interval 2001-2007 (see: Detection bias). In addition, DRSP is more expensive than the reference CHCs, and in some of the study centres DRSP users had to pay all or part of the costs (Dr Sidney, personal communication, 2012). Women who could afford to pay were better off, and on average better educated, more conscious of VTE risk, and more likely to be diagnosed if they developed VTE (see: Detection bias). And women who for one or another reason stopped using fully reimbursed preparations could later have re-started with DRSP.

Confounding could also have occurred because between 2001 and 2007 the prevalence of obesity increased, ${ }^{15}$ and over that interval the ratio of DRSP use to the use of the reference CHCs also increased.

Finally, the ostensibly detailed allowance made for multiple potential confounding factors was illusory. Apart from adjustment for age, site and calendar time, the potential confounding effect of 12 additional variables was also assessed: the latter variables were not included in the statistical models because they did not confound. However, factors such as ACE inhibitors, acne, $\beta$-blockers, hyperlipidaemia, hypertension, migraine, NSAIDs and statins are not risk factors for VTE. 
Statistical stability and strength of association

The overall RR estimate for DRSP users was 1.77 (95\% CI 1.33-2.35). Such a modestly elevated RR could readily have been due to bias or confounding, and among women aged 35-55 years the RR of 1.20 (95\% CI 0.78-1.84) could also have been due to chance (see: Internal consistency; Statistical stability and strength of association). In addition, the division of the data into the age categories 10-34 and 3555 years was arbitrary, and inadequate.

\section{Duration-response}

In an optimal comparison first-time-ever users of DRSP and of reference CHCs, who also commenced use at the same time, and who were the same age, should have been compared (see: Time order; Detection bias; Confounding). Or as a second-best option, first-time-ever-users together with re-starters who had not used a CHC for several years should have been compared (see: Time order). Closely similar durations of use should also have been compared, and with automated data it was not possible to do so, since it was not possible to precisely document the exact duration of $\mathrm{CHC}$ use, especially use that lasted only a few months. Moreover, since the newest CHC (DRSP) would on average have tended to be used for a shorter time than the older reference CHCs, the RR for DRSP users could have been overestimated.

There was quantitative evidence to indicate that the duration data were unreliable. Among the DRSP users, for $<3$ months of use versus $>12$ months of use, the incidence of VTE declined by 2.2-fold (from 12.8 to 5.7 per $10000 \mathrm{PY}$ ) (the authors' table 5), as would be expected. By contrast, however, there was hardly any decline among users of the reference CHCs: 1.2 -fold (from 9.3 to 7.8 per 10000 PY) (see: Internal consistency).

\section{Internal consistency}

As pointed out above the duration data were inconsistent (see: Duration-response).

Adjusted incidence rates of VTE in users of DRSP and in users of the reference CHCs were given in the published paper, ${ }^{3}$ and the unadjusted rates could be

Table 1 Unadjusted and adjusted* incidence rates of venous thromboembolism among users of drospirenone and users of reference combined hormonal contraceptives

\begin{tabular}{lcclll}
\hline & & \multicolumn{2}{l}{ Rate/10 $\mathbf{5}^{\mathbf{P Y}}$} \\
\cline { 3 - 5 } Contraceptive & Exposure (PY) & VTEs $(\boldsymbol{n})$ & Unadjusted & Adjusted \\
\hline DRSP & 80171 & 74 & 9.2 & 13.7 \\
Reference CHCS & 248031 & 205 & 8.3 & 8.2 \\
\hline
\end{tabular}

${ }^{*}$ Adjusted for age, site, and year of study entry.

CHC, combined hormonal contraceptive; DRSP, drospirenone; PY, person years; VTE, venous thromboembolism. calculated from the authors' table 3: for DRSP users the unadjusted and adjusted rates were 9.2 and 13.7 per 10000 PY, respectively (Table 1 ). Yet among users of the reference CHCs the unadjusted and adjusted rates were virtually unchanged: 8.3 and 8.2 per 10000 PY, respectively. The inconsistencies were minor, but explanations were not given.

The age-stratified RR estimates were also inconsistent: among women aged 10-34 years the RR was $2.21 \quad(95 \%$ CI 1.43-3.15), whereas among women aged $35-55$ years the RR was 1.20 (95\% CI $0.78-1.84$ ) (see: Statistical stability and strength of association). There is no biological mechanism that could explain an increased risk of VTE among DRSP users in one age group, but not in another (see: Biological plausibility).

\section{External consistency}

The authors stated ${ }^{3}$ that "compared to low-dose estrogen CHCs that have been on the market for longer periods of time ... the results [for DRSP users] have been mixed, with five [five is an error: the correct number is six] of eight studies [the correct number is nine] showing an increased risk of VTE ${ }^{21-26 ”,}$, and that "it is unclear whether the differences in findings arose from differences in study methodologies or differences in populations studied".

On the contrary, the reasons for the differences are abundantly clear: two of the three studies that did not identify an increased risk of VTE in DRSP users (one cohort study ${ }^{10}$ and one case-control study ${ }^{27}$ ) were designed to evaluate the specific hypothesis in detail. Complete information was obtained on confounders such as BMI and family history of VTE, and detailed provision was made to compare starters with starters and re-starters with re-starters, as well as to accurately record and compare similar durations of $\mathrm{CHC}$ use. The third study was a cohort study based on automated data in the USA ${ }^{28}$; that study was carried out before publicity had been given to the alleged increased risk of VTE in DRSP users. In all three studies the RRs approximated 1.0, and the data were sufficiently robust to document upper $95 \%$ CIs of $<2.0$.

By contrast, among the six studies showing an increased risk, one ${ }^{21}$ was a case-control study conducted for another purpose, it lacked adequate statistical power, and there was unambiguous evidence of detection bias. ${ }^{19}$ The remaining five studies were derived from automated data, detection bias was present in all five, in none of them were starters and re-starters properly defined and compared, in none of them was duration of use properly defined or allowed for, and in none of them was confounding adequately controlled. ${ }^{19}$ In addition, in three studies, ${ }^{23} 2426$ there was major under-ascertainment of cases of VTE, and in one study ${ }^{26}$ the diagnosis of VTE was not validated.

To sum up: studies specifically designed to make exact comparisons with full adjustment for confounding have 
not identified increased risks among DRSP users relative to users of reference CHCs. All but one of the six studies that have identified increased risks were derived from automated databases designed principally for administrative purposes, and they lacked essential information needed to carry out valid analyses. In addition, all six studies were methodologically defective.

\section{Biological plausibility}

There is no biologically plausible evidence to suggest that DRSP is more thrombogenic than other CHCs. DRSP increases sex-hormone-globulin-binding levels, but that effect has not been shown to be thrombogenic. $^{29}$ Some studies but not others ${ }^{12}{ }^{13}$ have reported changes in protein $\mathrm{C}$ metabolism, but again those changes have not been shown to be thrombogenic.

\section{ARTERIAL THROMBOEMBOLISM}

In this study ATE comprised a combined category of cases of MI and stroke, and there were 14 DRSP-exposed cases: the numbers of exposed cases with each disease were not given, but based on the overall distribution, at a guess about four or five could possibly have been $\mathrm{MI}$, and nine or 10 could have been stroke; among 11 DRSP users with ATE aged 35-55 years the numbers would have been smaller. On a priori grounds such sparse data are uninterpretable. Nevertheless, below I briefly apply causal criteria to the evidence.

\section{Time order}

Angina pectoris commonly precedes the onset of MI, and transient ischaemic attacks commonly precede the onset of stroke. If early symptoms of either disease selectively resulted in the use of DRSP, time order was violated (see: Detection bias; Confounding).

\section{Detection bias}

DRSP users experiencing chest pain could selectively have been investigated to rule out pulmonary embolism and instead have been found to have MI.

\section{Confounding}

Shared risk factors for MI and stroke include obesity, diabetes, family history, hypertension, smoking and socioeconomic status - although the magnitude of the risks of MI and stroke associated with each of the factors varies. Each of these factors could also have been determinants of DRSP use. In the database only clinically diagnosed diabetes was recorded, but was probably under-ascertained; and only data on some indirect and incomplete indicators of an increased risk of MI or stroke, such as ACE inhibitors, $\beta$-blockers, hyperlipidaemia, hypertension and statins were recorded. Major risk factors such as BMI, smoking, family history and socioeconomic status were not ascertained.
DRSP is an aldosterone antagonist, and it reduces blood pressure $;^{20}$ its use also results in water loss, and since the weight gain caused by other $\mathrm{CHCs}$ is avoided, it tends selectively to be used by obese women ${ }^{10}$ (see: Confounding; Biological plausibility).

There were 14 DRSP-exposed cases of ATE, and multivariate adjustment was made for one continuous variable (age) and five categorical variables (site, year of entry, hypertension, hyperlipidaemia, diabetes). Not only were hypertension, hyperlipidaemia and diabetes under-ascertained, but with only 14 cases of ATE simultaneous adjustment for variables that could take on a total of 17 values (age, 2; site, 4; year of entry, 7; hypertension, 2; hyperlipidaemia, 2) and 224 value combinations, was virtually meaningless (see: Statistical stability and strength of association).

\section{Statistical stability and strength of association}

The lower 95\% CI for the overall RR of 2.01 was 1.06 , indicating that the data were fragile, and susceptible to the vagaries of error: had there been 13 instead of 14 DRSP-exposed cases the lower CI would have included 1.00, and by conventional standards the association could have been due to chance. The additional claim that the association was confined to women over the age of 35 years was arbitrary, and it was biologically implausible (see: Internal consistency; Biological plausibility). And still further, because of limited numbers an evaluation of statistical stability within relevant strata (e.g. half-decade or decade of age) was not feasible. And as pointed out above, the data were so sparse that multivariate adjustment for confounding was virtually meaningless (see: Confounding).

\section{Duration-response}

The data were too sparse for an evaluation of duration-response to be feasible.

\section{Internal consistency}

The data were too sparse for an evaluation of internal consistency within strata such as half-decade or decade of age to be feasible. Information on consistency in other relevant strata, such as BMI, was missing (see: Confounding).

\section{External consistency}

Evidence in other studies suggests that DRSP does not increase the risk of ATE. ${ }^{30}$ It is established that DRSP reduces blood pressure ${ }^{20}$ (see: Biological plausibility), and there is evidence of a reduction in the risk of ATE in menopausal women using DRSP as hormone replacement therapy. ${ }^{30}$

\section{Biological plausibility}

The use of CHCs, combined with heavy smoking, increases the risk of $\mathrm{MI}^{31}$; $\mathrm{CHCs}$ also increase the risk of stroke. ${ }^{32}$ However, there is no experimental, pathological or epidemiological evidence to suggest that 
DRSP increases the risk of ATE more than do other CHCs. If anything, the antihypertensive effects of $\mathrm{DRSP}^{20}$ coupled with the existing epidemiological evidence $^{30}$ (see: External consistency), suggest that DRSP reduces the risk of ATE, relative to other CHCs.

\section{MORTALITY}

Total mortality was not increased in DRSP users. What is relevant, however, is cardiovascular mortality, the risk of which, given the purported increased risks of VTE and ATE, should also have been increased. In the published report ${ }^{3}$ data on cardiovascular mortality were not provided, but they were provided on the FDA website (table 10c) ${ }^{1}$ : among new users of DRSP there was one cardiovascular death, and among users of the reference CHCs there were 15 (RR, 0.17; 95\% CI 0.02-1.35). The reduction in the risk of cardiovascular death, instead of an increase, supports the likelihood that the analyses of VTE and ATE risk in DRSP users were biased and confounded.

\section{CONCLUSIONS}

\section{Venous thromboembolism}

The findings in this study did not satisfy the criteria of time order, detection bias, confounding, statistical stability and strength of association, durationresponse, internal consistency, external consistency or biological plausibility.

What remains to be explained is why so many unsatisfactory studies, particularly studies derived from large databases containing incomplete and imprecise data assembled for administrative purposes, and sharing much the same biases and confounders, have been conducted. Why the issues have become contentious, and sometimes even ad hominem ${ }^{19}$ also needs to be explained.

The origins of the contentiousness date back to the 1990 s, and to the claim that so-called 'third-generation' CHCs more commonly cause VTE than do 'secondgeneration' CHCs. ${ }^{12} 13$ That claim originated in a study sponsored by the World Health Organization, ${ }^{33}$ following which there was a spate of studies, some confirming the existence of an association, and some rebutting it. $^{34-36}$ It appears that the controversy has now spilled over to DRSP, which has been designated as a 'fourthgeneration' $\mathrm{CHC}$. There is no scientific rationale underlying the 'fourth-generation' designation.

The best evidence continues to suggest that the risk of VTE is not greater for users of DRSP than for users of other CHCS. The most rigorously conducted studies continue to demonstrate that the risk of VTE is a class effect of all CHCs, and that the magnitude of the risk is dependent on the dose of $\mathrm{EE}$, and independent of the specific progestogen contained in the pill.

\section{Arterial thromboembolism}

The findings for this outcome failed to satisfy any of the epidemiological principles of causation.

\section{Cardiovascular mortality}

There was only one cardiovascular death in DRSP users, and none of the criteria of causation were satisfied.

Competing interests The author presently consults, and in the past has consulted, with Bayer AG, the manufacturer of drospirenone, and with manufacturers of other combined hormonal contraceptives mentioned in this article.

Provenance and peer review Not commissioned; externally peer reviewed.

\section{REFERENCES}

1 Ouellet-Hellstrom R, Graham DJ, Staffa JA, et al. Combined Hormonal Contraceptives (CHCs) and the Risk of Cardiovascular Disease Endpoints. CHC-CVD final report 111022v2. FDA website 2011. http://www.fda.gov/Drugs/DrugSafety/ucm277346. htm [accessed 3 February 2013].

2 Lidegaard Ø, Milsom I, Geirsson RT, et al. Hormonal contraception and venous thrombosis. Acta Obstet Gynecol Scand 2012;91:769-778.

3 Sidney S, Cheetham TC, Connell FA, et al. Recent combined hormonal contraceptives (CHCs) and risk of thromboembolism and other cardiovascular events in new users. Contraception 2012;dx.doi.org/10.1016/.contraception.2012.09.015 [accessed 3 February 2013].

4 US Department of Health, Education, and Welfare: Public Health Service. Smoking and Health: Report of the Advisory Committee to the Surgeon General of the Public Health Service (Public Health Service Publication No. 11030). Washington, DC: U.S. Government Printing Office, 1964.

5 Hill AB. The environment and disease: association or causation. Proc Royal Soc Med 1965;58:295-300.

6 Susser M. Causal Thinking in the Health Sciences: Concepts and Strategies of Epidemiology. New York, NY: Oxford University Press, 1973.

7 Susser M. What is a cause and how do we know one: a grammar for pragmatic epidemiology. Am J Epidemiol 1991;133:635-658.

8 Medical Research Council Working Group. Risk of thromboembolic disease in women taking oral contraceptives. BMJ 1967;2:355-359.

9 White RH. The epidemiology of venous thromboembolism. Circulation 2003;107:I4-I8.

10 Dinger JC, Heinemann LA, Kühl-Habich D. The safety of a drospirenone-containing oral contraceptive: final results from the European Active Surveillance study on oral contraceptives based on 142,475 women-years of observation. Contraception 2007;75:344-354.

11 Rabe T, Luxembourg B, Ludwig M, et al. Contraception and thrombophilia - a statement from the German Society of Endocrinology and Reproductive Medicine (DGEF e.v) and the Professionals Asssociation of Germn Gynaecologistis. J Reprod Endocrinol 2011;8:126-167.

12 Reid RL, Westhoff C, Mansour D, et al. Oral contraceptives and venous thromboembolism: consensus opinion from an international workshop held in Berlin, Germany in December 2009. J Fam Plann Reprod Health Care 2010;36:117-122.

13 Reid RL. Oral contraceptives and venous thromboembolism: pill scares and public health. J Obstet Gynaecol Can 2011;33:1150-1155.

14 Heinemann K, Heinemann LA. Comparative risks of venous thromboembolism among users of oral contraceptives 
containing drospirenone and levonorgestrel. J Fam Plann Reprod Health Care 2011;37:132-135.

15 Ogden CL, Carroll MD, Kit BK, et al. Prevalence of obesity in the United States, 2009-2010. NCHS Data Brief 2012; 1-8. http://www.cdc.gov/obesity/data/adult.html [accessed 3 February 2013].

16 Shapiro S, Rosenberg L. Bias in case-control studies. In: Armitage P, Colton T (eds), Encyclopedia of Biostatistics, Vol 1. Chichester, UK: John Wiley \& Sons, 1998;313-322.

17 Shapiro S. Bias in the evaluation of low-magnitude associations: an empirical perspective. Am J Epidemiol 2000;151:939-945.

18 Dinger J. Oral contraceptives and venous thromboembolism: old questions revisited. J Fam Plann Reprod Health Care 2009;35:211-213.

19 Dinger J, Shapiro S. Combined oral contraceptives, venous thromboembolism, and the problem of interpreting large but incomplete datasets. J Fam Plann Reprod Health Care 2012;38:2-6.

20 Archer DF, Thorneycroft IH, Foegh M, et al. Long-term safety of drospirenone-estradiol for hormone therapy: a randomized, double-blind, multicenter trial. Menopause 2005;12:716-727.

21 van Hycklama Vlieg A, Helmerhorst FM, Vandenbroucke JP, et al. The venous thrombotic risk of oral contraceptives; effects of estrogen dose and progestogen type: results of the MEGA case-control study. BMJ 2009;339:b2921.

22 Lidegaard Ø, Løkkegaard E, Svendsen AL, et al. Hormonal contraception and risk of venous thromboembolism: national follow-up study. BMJ 2009;339:b2890.

23 Parkin L, Sharples K, Hernandez RK, et al. Risk of venous thromboembolism in users of oral contraceptives containing drospirenone or levonorgestrel: nested case-control study based on UK General Practice Research Database. BMJ 2011;342:d2139.

24 Jick SS, Hernandez RK. Risk of non-fatal venous thromboembolism in women using oral contraceptives containing drospirenone compared with women using oral contraceptives containing levonorgestrel: case-control study using United States claims data. BMJ 2011;342:d2151.

25 Lidegaard Ø, Nielsen LH, Skovlund CW, et al. Risk of venous thromboembolism from use of oral contraceptives containing different progestogens and oestrogen doses: Danish cohort study, 2001-9. BMJ 2011;343:d6423.
26 Gronich N, Lavi I, Rennert G. Higher risk of venous thrombosis associated with drospirenone-containing oral contraceptives: a population-based cohort study. CMAJ 2011;183:E1319-E1325.

27 Dinger J, Assmann A, Möhner S, et al. Risk of venous thromboembolism and the use of dienogest- and drospirenone-containing oral contraceptives: results from a German case-control study. J Fam Plann Reprod Health Care 2010;36:123-129.

28 Seeger JD, Loughlin J, Eng PM, et al. Risk of thromboembolism in women taking ethinylestradiol/ drospirenone and other oral contraceptives. Obstet Gynecol 2007;110:587-593.

29 Stanczyk FZ, Grimes DA. Sex hormone-binding globulin: not a surrogate marker for venous thromboembolism in women using oral contraceptives. Contraception 2008;78:201-203.

30 Assmann A, Heinemann K, Dinger J. The safety of oral hormone replacement therapy: final results from the EURAS-HRT study (Abstract). Pharmacoepidemiol Drug Saf 2011;29:S181.

31 Slone D, Shapiro S, Rosenberg L, et al. Relation of cigarette smoking to myocardial infarction in young women. $\mathrm{N} \mathrm{Engl} \mathrm{J}$ Med 1978;298:1273-1276.

32 Collaborative Group for the Study of Stroke in Young Women. Oral contraception and increased risk of cerebral ischemia or thrombosis. N Engl J Med 1973;288:871-878.

33 World Health Organization Collaborative Study of Cardiovascular Disease and Steroid Hormone Contraception. Effect of different progestagens in low oestrogen oral contraceptives on venous thromboembolic disease. Lancet 1995;346:1582-1588.

34 Lewis MA, Heinemann LA, MacRae KD, et al. The increased risk of venous thromboembolism and the use of third generation progestagens: role of bias in observational research. The Transnational Research Group on Oral Contraceptives and the Health of Young Women. Contraception 1996;54:5-13.

35 Lewis MA, MacRae KD, Kühl-Habich D, et al. The differential risk of oral contraceptives: the impact of full exposure history. Hum Reprod 1999;14:1493-1499.

36 Shapiro S, Dinger J. Risk of VTE among users of oral contraceptives. J Fam Plann Reprod Health Care 2010;36:103.

\section{FACULTY AWARDS}

The Faculty of Sexual and Reproductive Healthcare has available a number of annual awards for which applications are invited from Faculty members and non-members as listed below. Details of the individual awards, together with an application form and/or guidelines on how to apply and any eligibility criteria, may be found on the Faculty website at www.fsrh.org.

\section{The 4-0-8 Sheffield Fund}

Award: Approximately $£ 1000$ will be allocated every 3 months, either as a single award or divided between the successful applicants, for the purpose of funding Educational Training for health care professionals who have limited funding for training.

Eligibility: Individuals (undergraduate medical students)

Closing date: See website for details

\section{Margaret Pyke Trust Prize Oral Presentation}

Award: This prize is awarded annually within the Annual Scientifi c Meeting for the best oral presentation out of the fi ve selected from the abstracts submitted. The dates and deadlines do vary but are publicised both on the Faculty website, from January in the relevant year, and within the conference publicity.

Eligibility: Individuals (Faculty members) or teams

Closing date: 7 April annually 